age was $32 \mathrm{y}$, median number of sexual partners in last 3 months was 4 . Reported drug use in the previous month was 38\%; 19\% had injected drugs. Syphilis stage was primary (31\%), secondary (25\%), early latent (45\%).Up to February 2016, total follow-up was 144 person-years. 12 (11\%) were newly diagnosed HIV-positive. HIV incidence was 8.3 (95\% confidence interval, CI 4.214) per 100 person-years follow-up (HPYFU). Incidence of rectal STIs was: rectal chlamydia, 27 HPYFU (CI 19-36); rectal gonorrhoea, 33 HPYFU (CI 25-44); syphilis re-infection, 10 HPYFU (CI 5.7-17).

Conclusions. The significant risk of HIV seroconversion following a diagnosis of early syphilis suggests that this group may particularly benefit from the use of pre-exposure prophylaxis. The high levels of subsequent rectal infections support the inclusion of regular STI screening in PrEP management guidelines.

\section{P173 MULTI-DRUG USE, AND ASSOCIATED FACTORS, WITHIN A COMMUNITY BASED SAMPLE OF GAY AND BISEXUAL MEN IN SCOTLAND}

Julie Riddell ${ }^{*}$, Nicola Boydell, Lisa McDaid. MRC/CSO Social and Public Health Sciences Unit, Glasgow, UK

\subsection{6/sextrans-2016-052718.223}

Background/introduction Research suggests that drug use may be higher among men who have sex with men (MSM), and links between drug use and risky sexual behaviours are well established. Evidence suggests that MSM who report 'ever using' drugs may report using a variety of drug types.

Aim(s)/objectives To explore multi-drug use and associated sexual risk behaviours within a community sample of MSM in Scotland.

Methods Analysis of data from 1292 MSM participating in barbased surveys in Scotland in 2014. Factors related to 'ever using' and multi-drug use within the previous 12 months were examined. Multi-drug use was calculated using those who reported more than one type of drug use in the previous 12 months (injecting, snorting or psychoactive drugs).

Results The mean age of men sampled was 34.72 years (range $18-82$, SD $=11.23)$. Most men identified as gay $(92.3 \%)$ and reported being educated post 16 (85.8\%). 42.6\% of men reported 'ever using' drugs and of those, $55.3 \%$ had used within the last 12 months. $47.8 \%$ of men using within the last 12 months reported multi-drug use. Men who had used drugs within the last 12 months and those reporting multi-drug use were more likely to report more risky sex, specifically group sex. However, they also reported higher rates of both HIV and STI testing.

Discussion/conclusion The percentage of men reporting multidrug use is consistent with previous research. Although men reported more risky sexual behaviours, testing rates would suggest they understand and respond to these risks. HIV/STI screening may present opportunities to identify and address potentially problematic drug use with attendees.

\section{P174 MANCHESTER CHEMSEX - INJECTING STRAIGHT UP THE M6}

${ }^{1}$ Chris Ward*, 'Debbie Thomas, ${ }^{2,1}$ Terri Anderson, ${ }^{1}$ Rebecca Evans, ${ }^{1}$ Orla McQuillan. ${ }^{1}$ Central Manchester University Hospitals NHS Foundation Trust, Manchester, UK; ${ }^{2}$ Addiction Dependency Solutions, Manchester, UK
Background/introduction Chemsex involves sex under the influence of psychoactive drugs such as gamma-hydroxybutyric acid (GHB), mephedrone and crystal methamphetamine. There's been an increasing trend of chemsex use among UK men who have sex with men (MSM) requiring services to tackle this growing problem.

Aim(s)/objectives To review the first year's progress of a newly developed chemsex clinic co-commissioned by sexual health and drugs and alcohol commissioners.

Methods Prospective data collection of patients attending clinic from April 2015 to March 2016. Data was collected on demographics, risk taking, sexually transmitted infections (STIs) and drug use.

Results 43 patients were seen. 34 (79.1\%) were White British, and $42(97.7 \%)$ were MSM. 19 (44.2\%) were referred through genitourinary medicine (GUM) clinics, of which 10 (52.6\%) were for post-exposure prophylaxis, 9 (20.9\%) through HIV services, and 7 (16.3\%) self-referrals. 24 (55.8\%) were HIV positive, and $10(23.3 \%)$ had Hepatitis C. 32 (74.4\%) reported episodes of condomless sex, $21(48.8 \%)$ engaged regularly in group sex and 5 (11.9\%) participated in fisting, highlighting high rates of sexual risk taking. $36(83.7 \%)$ patients reported taking mephedrone, 29 (67.4\%) GHB, and 12 (27.9\%) crystal meth. 23 $(53.5 \%)$ patients injected. 21 STIs were found in $16(37.2 \%)$ patients, with 10 (47.6\%) Gonorrhoea infections, 4 (19.0\%) chlamydia, 3 (14.3\%) syphilis and 3 (14.3\%) Hepatitis C.

Discussion/conclusion Our data shows high rates of risk taking among chemsex participants with resultant high rates of STIs. Targeted harm reduction interventions need to be developed in GUM clinics to continue to address this issue.

\section{P175 SERVICE EVALUATION OF THE USE OF THE YOUNG PERSON'S PROFORMA IN RELATION TO CENTRAL AND COMMUNITY SEXUAL HEALTH CLINICS}

${ }^{1}$ Megan O'Connell, ${ }^{2}$ Nicola Lomax*. ${ }^{1}$ Cardiff University, Cardiff, UK; ${ }^{2}$ Cardiff Royal Infirmary, Cardiff, UK

\subsection{6/sextrans-2016-052718.225}

Background/introduction The prevalence of non-consensual sex is higher in those with a sexual debut before the age of 16 . BASHH therefore advocates the use of a Young Person's Proforma (YPP) in sexual health clinics to detect signs of, and concurrent risk factors for, child sexual exploitation (CSE), intending to safeguard this vulnerable group of attendees.

Aim(s)/objectives To investigate the adherence to BASHH guidelines relating to the care of young people accessing sexual health services, specifically the use of the YPP.

Methods A retrospective review analysing 150 case notes of patients under $16 y r$, attending between $1^{\text {st }}$ July 2014 and $1^{\text {st }}$ June 2015. Notes were extracted from a central clinic $(\mathrm{n}=50)$, and 4 community sexual health clinics $(\mathrm{n}=100)$.

Results Centrally, all patients had a proforma completed, compared with $81 \%$ of community patients. Proformas were completed in $67 \%$ of male community patients. 19 patients had experienced involuntary sexual activity. $19 \%$ of patients had 1 or more significant risk factor for CSE. In total, 83 further referrals (57\% safeguarding) were made. Risk factors were reassessed in $79 \%$ of patients. $71 \%$ attended primarily for contraception (central $=46 \%$, community $=83 \%$ ), with $79 \%$ offered STI screening. 